\title{
Pathophysiologie, Diagnostik und Therapie der chronischen metabolischen Azidose bei Niereninsuffizienz
}

Der Begriff Azidose wird bei diabetischen Patienten meist mit der akuten Ketoazidose als Ausdruck eines absoluten Insulinmangels assoziiert. Weit weniger im diagnostischen Fokus steht die chronische metabolische Azidose, die durch eine langfristige Erschöpfung des Bikarbonatpuffers im Blut charakterisiert ist. Ursache der chronischen metabolischen Azidose ist in der überwiegenden Zahl der Fälle ein Funktionsverlust der Niere, der zu einer Störung des BikarbonatPuffersystems führt. Dieses Puffersystem ist für etwa 75\% der Gesamt-Pufferkapazität des Blutes verantwortlich und als offenes System in der Lage, auch einen stark erhöhten Anfall von Säureäquivalenten, z.B. durch intensive Muskelaktivität, abzufangen. Die Pufferung der Säureäquivalente erfolgt durch das Bikarbonat-Ion $\left(\mathrm{HCO}_{3-}\right)$, das nach Bindung eines Säureäquivalents (Proton, $\mathrm{H}^{+}$) in Kohlendioxid $\left(\mathrm{CO}_{2}\right)$ und Wasser $\left(\mathrm{H}_{2} \mathrm{O}\right)$ zerfällt. Das Kohlendioxid wird über die Lunge abgeatmet und so Säureäquivalente entsorgt (Abb. 2). Doch dieses System funktioniert nur, wenn die Niere ausreichend Hydrogenkarbonat-Ionen rückresorbieren kann. Daher verliert das Blut Pufferkapazität, wenn die Nierenfunktion nachlässt.

Diabetes mellitus ist wiederum die häufigste Ursache für Nierenschäden bis hin zur terminalen Niereninsuffizienz [1, 2]: 20-40\% der Diabetespatienten entwickeln im Krankheitsverlauf eine Nierenerkrankung (diabetische Nephropathie) [3]; bei $28 \%$ der prävalenten Fälle und bei $34 \%$ der jährlich hinzukommenden Fälle terminaler Niereninsuffizienz wird Dia-



Abb. 1 Hypothetische Wechselwirkungen zwischen chronischer metabolischer Azidose, Diabetes mellitus und chronischer Niereninsuffizienz.

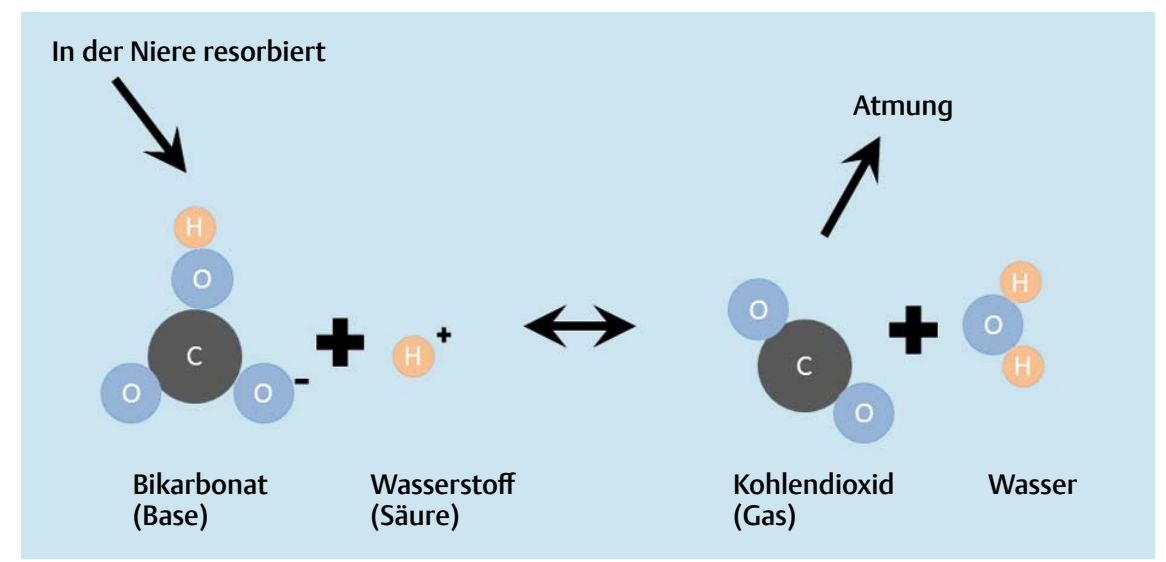

Abb. 2 Bikarbonat-Puffersystem.

betes mellitus als Grunderkrankung diagnostiziert [1]. Inzwischen stellen Diabetiker die Hälfte aller Patienten, die erstmals eine Dialyse erhalten. Ursächlich für die diabetische Nephropathie scheint die Hyperglykämie zu sein, die in den Glomeruli zu einer Verdickung der Basalmembran, einer Verbreiterung des Mesangiums und einer Sklerosierung führt [4]. Dies verursacht wiederum Permeabilitätsstörungen. Es entsteht zunächst eine Mikroalbuminurie und mit abnehmender Nierenfunktion eine Makroalbuminurie und eine Erhöhung des Serumkreatinins, bis letztendlich eine Nierenersatztherapie notwendig wird [5].

Die Niereninsuffizienz wiederum führt unter anderem zu einer Störung des Säuren-Basen-Gleichgewichts. Die Niere kann nicht mehr genug Säureäquivalente ausscheiden und Bikarbonat produzieren, wodurch der Bikarbonatspiegel im Plasma sinkt [6]. Die daraus resultierende chronische metabolische Azidose fördert die Progression der Niereninsuffizienz [7, 8] (Abb. 1).

\section{Zusammenhang zwischen \\ Niereninsuffizienz und chronischer metabolischer Azidose}

Eine Auswertung der Nurses' Health Study fand, dass der Bikarbonatspiegel mit dem Diabetesrisiko korreliert. Frauen mit Bikarbonatspiegeln über dem Median der Gesamtpopulation $(22,4 \mathrm{mmol} / \mathrm{l})$ hatten ein $24 \%$ geringeres Risiko, innerhalb von 10 Jahren einen Typ-2-Diabetes zu entwickeln. Dieser Zusammenhang bestand auch nach der Adjustierung für die Ernährung der Probandinnen. Die Autoren vermuten, dass eine metabolische Azidose die Insulinresistenz fördert und dadurch das Risiko eines Diabetes erhöht [1].

Falls diese Hypothese bestätigt werden kann, besteht ein doppelter Teufelskreis zwischen metabolischer Azidose, Diabetes und chronischer Niereninsuffizienz (CKD): Ein niedriger Bikarbonatspiegel fördert über die Ausbildung einer Insulinresistenz die Entwicklung eines Diabetes (Abb. 2).

Eine weitere klinische Folge sowohl der Niereninsuffizienz als auch des Diabetes ist die Muskelkatabolie. Möglicherweise wird dies durch eine Insulinresistenz verursacht: Insulin erhält die Muskelmasse, indem es die Proteolyse unterdrückt [911]. Bei einem Insulinmangel ist dagegen die Proteolyse im Muskel beschleunigt $[12,13]$. Durch eine verminderte Reaktion auf Insulin oder auch auf IGF-1 (Insulinähnlicher Wachstumsfaktor) wird u.a. ein Signalweg (Ubiquitin/ProteasomWeg) im Muskel aktiviert, der den Abbau von Muskeleiweiß stimuliert [14]. Die chronische metabolische Azidose aktiviert ebenfalls den Proteinabbau. Sie führt vermutlich durch die Störung von Signalwegen aufgrund azidotisch bedingter Enzymdegradation, eine IGF-1-Dysregulation, Insulinresistenz und Entzündungsreaktionen zu einem Muskelabbau [15-19]. Eine wichtige Rolle scheint ein gestörter Signalweg (PI3K/Akt) zu spielen, der normalerweise die anabolen und antikatabolen Effekte von IGF-1 und Insu- 
Tab. 1 Normalbereiche der Blutgasanalyse [21].

\begin{tabular}{ll}
\hline \multicolumn{2}{l}{ Normalbereiche der Blutgasanalyse (BGA) } \\
\hline $\mathrm{pH}-$ Wert & $7,36-7,44$ \\
\hline $\mathrm{PO}_{2}$ (Sauerstoffpartialdruck) & $75-100 \mathrm{mmHg}$ \\
\hline $\begin{array}{l}\mathrm{PCO}_{2} \text { (Kohlendioxidpartial- } \\
\text { druck, arteriell) }\end{array}$ & $35-45 \mathrm{mmHg}$ \\
\hline $\begin{array}{l}\text { Standard-Bikarbonat } \\
\text { (arteriell) }\end{array}$ & $22-26 \mathrm{mmol} / \mathrm{l}$ \\
\hline $\begin{array}{l}\text { Basenüberschuss (BE) } \\
\mathrm{O}_{2} \text {-Sättigung }\end{array}$ & $-2-+2 \mathrm{mmol} / \mathrm{l}$ \\
\hline
\end{tabular}

lin vermittelt [20]. Daher wird durch das Zusammentreffen von Niereninsuffizienz, Diabetes und chronischer metabolischer Azidose über die Entwicklung einer Insulinresistenz die Muskelkatabolie möglicherweise verstärkt.

\section{Diagnostik und Therapie der}

chronischen metabolischen Azidose

Die chronische metabolische Azidose wird über eine Blutgasanalyse diagnostiziert (Tab. 1). Dabei sind in erster Linie der pH-Wert, der Bikarbonatspiegel $\left(\mathrm{HCO}_{3}\right)$ und Basenüberschuss (Base-Excess, BE) von Bedeutung. Der pH-Wert des Blutes sollte zwischen 7,36 und 7,44 liegen, der Bikarbonatspiegel liegt idealerweise zwischen 22 und $26 \mathrm{mmol} / \mathrm{l}$ und der Normwert des BE liegt zwischen -2 und +2 . Über die Anionenlücke kann die chronische metabolische Azidose von anderen Azidoseformen abgegrenzt werden. Hierbei werden die nicht erfassten Anionen im Plasma berechnet, die je nach Art der metabolischen Azidose unterschiedlich beeinflusst werden (Tab. 1).

Bereits durch eine angepasste Ernährung kann der Bikarbonatspiegel angehoben werden. Eine Studie mit Hypertonikern in CKD-Stadium 4 konnte zeigen, dass eine obst- und gemüsereiche Ernährung innerhalb eines Jahres den Bikarbonatspiegel genauso effektiv erhöht wie eine Behandlung mit oralem Natriumbikarbonat [22] Die Aussagekraft dieser Studie für die Umsetzung in die ärztliche Praxis wird allerdings dadurch eingeschränkt, dass die Patienten ihre vegetarische Supplementation kostenfrei erhielten. In der Praxis wird daher die mangelnde Compliance der Patienten diesem Vorgehen Grenzen setzen. Die Einnahme von oralem Bikarbonat alleine oder idealerweise ergänzend zu einer Anpassung der Ernährungsgewohnheiten bietet eine zuverlässigere Möglichkeit, der chronischen metabolischen Azidose und deren weitreichenden Folgen entgegenzu-

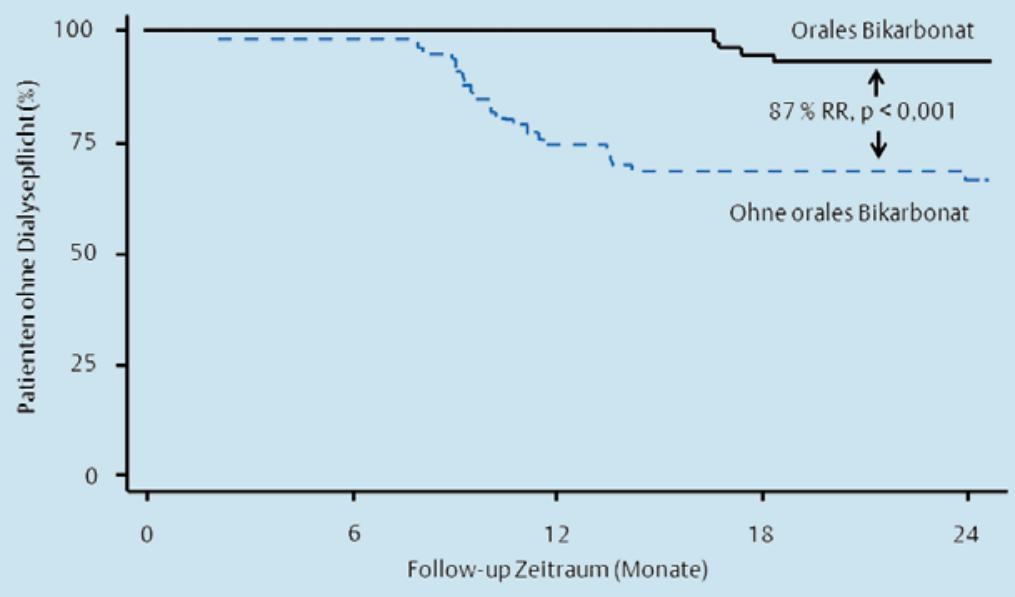

Abb. 3 Orales Bicarbonat über 2 Jahre senkte das relative Risiko (RR), dialysepflichtig zu werden, signifikant um $87 \%$ gegenüber Patienten ohne Bikarbonatsubstitution [23].

wirken. So kann der doppelte Teufelskreis durchbrochen und die weitere Progression der Niereninsuffizienz verlangsamt werden [23-25] (Abb. 3). Auch der Proteinkatabolismus kann durch die Therapie mit oralem Bikarbonat reduziert werden [26, 27]. Orales Bikarbonat erhöht effektiv und leitliniengerecht den Bikarbonatspiegel und ist dabei gut verträglich und einfach anwendbar [28]. Die Anzahl der benötigten Tabletten hält sich mit 2-4 pro Tag in einem von den meisten Patienten gut akzeptierten Bereich. Magensaftresistente Präparate sollten bevorzugt werden, da diese besser verträglich sind und die Aufnahme von Nährstoffen nicht durch eine Erhöhung des pH-Werts im Magen beeinflussen [28].

Durch die Korrektur des Bikarbonatspiegels können einige Konsequenzen der Niereninsuffizienz einfach und kostengünstig verhindert oder zumindest abgeschwächt werden, um die Lebensqualität der Patienten zu erhöhen. Magensaftresistentes orales Bikarbonat ist verordnungsfähig zur Behandlung bei chronischer Niereninsuffizienz. Aufgrund der Preisunterschiede bei magensaftresistenten BikarbonatPräparaten sollte jedoch ein Preisvergleich durchgeführt werden.

Aufgrund der pathophysiologischen Zusammenhänge zwischen Diabetes, Niereninsuffizienz und chronischer metabolischer Azidose sollte bei unklarer Allgemeinsymptomatik und Niereninsuffizienz auch an eine Überprüfung des Säure-Base-Gleichgewichts mittels Blutgasanalyse gedacht werden. Die Therapie kann durch Anpassung der Ernährung und durch magensaftresistentes orales Bikarbonat erfolgen. Dr. Marcel Kaiser, Frankfurt
Literatur

$1 \quad$ Frei $U$ et al. QuasiNiere 2008

2 Neufang-Sahr A, Scherbaum W. URL: http:// www.diabetes-deutschland.de/archiv/archiv_2382.htm [Stand: 27.02.13]

3 Bundesärztekammer (BÄK) Arbeitsgemeinschaft der Deutschen Ärztekammern et al. Nationale VersorgungsLeitlinie Nierenerkrankungen bei Diabetes im Erwachsenenalter. Version der Deutschen Diabetes Gesellschaft 2010

4 Dikow R. Dtsch Arztebl 2003; 100: 11001101

5 Adler Al et al. Kidney Int 2003; 63: 225-232

6 Keller CK et al. Praxis der Nephrologie. $3^{\text {rd }}$ ed. Berlin, New York: Springer 2010

7 Shah SN et al. AJKD 2009; 54: 270-277

8 Raphael KL et al. Kidney Int 2011; 79: 356362

9 Fryburg DA et al. J Clin Invest 1995; 96: 1722-1729

10 Grizard J et al. Reprod Nutr Dev 1999; 39: $61-74$

11 Kettelhut IC et al. Diabetes Metab Rev 1988; 4: 751-772

12 Mitch WE et al. Am J Physiol 1999; 276: C1132-8

13 Price SR et al. J Clin Invest 1996; 98: 17031708

14 Wang X. Endocrinology 2006; 147: 41604168

15 Bailey JL et al. J Clin Invest 1996; 97: 14471453

16 Bailey JL. JASN 2006; 17: 1388-1394

17 Ding H et al. J Clin Invest 1996; 97: 10641075

18 Fouque D et al. Kidney Int 1995; 47: 876883

19 Pickering WP et al. Kidney Int 2002; 61: 1286-1292

20 Heszele, Maria F Chacon et al. Endocrinology 2004; 145: 4803-4805

21 Dr. med. Olav Hagemann. URL: www.medweb24.de [Stand: 19.00.2014]

22 Goraya $N$ et al. CJASN 2013: 1-11

23 Brito-Ashurst I de et al. J Am Soc Nephrol 2009; 20: 2075-2084

24 Kovesdy CP. NDT 2012; 27: 3056-3062

25 Susantitaphong P et al. AJN 2012; 35: 540547

26 Abramowitz M et al. CJASN 2013

27 Verove C et al. J Ren Nutr 2002; 12: 224228

28 Breitkreutz J et al. JPP 2007; 59: 59-65 